\title{
Multiple-Channel Optimized Quantizers for Rayleigh Fading Channels
}

\author{
Yugang Zhou, Wai-Yip Chan, and Tiago H. Falk \\ Department of Electrical and Computer Engineering \\ Queen's University, Kingston, Ontario, Canada, K7L 3N6 \\ Email: \{zhouy,chan,falkt\}@ee.queensu.ca
}

\begin{abstract}
We consider multiple description communication over Rayleigh fading channels with binary phaseshift keying (BPSK) modulators at the transmitter and soft-decision detectors at the receiver. The multiplechannel optimized quantizer design (MCOQD) method, introduced in [7] for multiple discrete memoryless channels, is extended to multiple Rayleigh fading channels. The decision thresholds of the soft-decision detectors are optimized to achieve minimum end-to-end distortion. Simulation results show that MCOQD provides more robust quantizers than multiple description scalar quantizers [3] over Rayleigh fading channels, when both the encoder and decoder are matched to channel statistics, and when only the decoder is matched to channel statistics.
\end{abstract}

Keywords: Multiple description coding, joint sourcechannel coding, quantization, channel optimized quantizers, coding for erasure channels.

\section{INTRODUCTION}

Multiple description coding (MDC) is concerned with encoding information from a source into two or more descriptions, such that any subset of the descriptions can be used for source reconstruction, and the reconstruction quality improves with the size of the subset. The basic motivation for MDC is to provide reconstruction quality that degrades gracefully with the number of descriptions lost in transmission, e.g., over a packet network. An accessible introduction to MDC is provided by Goyal [1].

Most existing multiple description coding (MDC) schemes [3], [4] consider only "on-off" channels and are not suited to channels with symbol or bit errors. In [3], Vaishampayan introduced a technique, herein referred to as multiple-description scalar quantization (MDSQ), for designing scalar quantizers for two-description coding. The design procedure entails first selecting an index assignment (IA) and then optimizing the quantizer partition and reconstructions for the chosen IA. In [7], we proposed multiple channel optimized quantizer design
(MCOQD) and showed that the designed quantizers outperformed the multiple description scalar quantizers of [3] over discrete memoryless channels (DMCs) with symbol errors and erasures.

Yang et al. [5] applied the MDSQ in [3] to Rayleigh fading channels, where the channel was declared to be in a working state or erasure state by comparing a fading parameter with a predetermined threshold. An upper bound, instead of a closed form, of the channel erasure probability was derived. However, they discovered that the upper bound was too loose at low channel signal-tonoise-ratio (SNR) to be used for MDSQ design.

In this paper, the MCOQD in [7] is extended to Rayleigh fading channels with binary phase-shift keying (BPSK) modulators at the transmitter and soft-decision detectors at the receiver. The detectors are initially set to have three detection regions. Channel erasure is declared when the energy of the received signal is small (as determined by detector thresholds). Closed forms of the channel erasure and bit error probability are used for MCOQD. The soft detectors are then extended to have more than three detection regions. The decision thresholds are optimized to achieve minimum end-to-end distortion. Performance results are also provided for the case where the receiver but not the transmitter has perfect knowledge of channel statistics.

\section{REVIEW OF MCOQD FOR DMCS}

Channel optimized quantizer design (COQD) as originally proposed by Farvardin and Vaishampayan [2] is a joint source channel coding scheme for bit-error channels. With COQD, a scalar quantizer is designed to minimize the reconstruction error due to both quantization and channel errors. MCOQD is obtained by extending COQD to the case of sending two descriptions over two parallel channels, where both erasure and symbol errors may occur. Extension to more than two channels will be seen to be straightforward. 


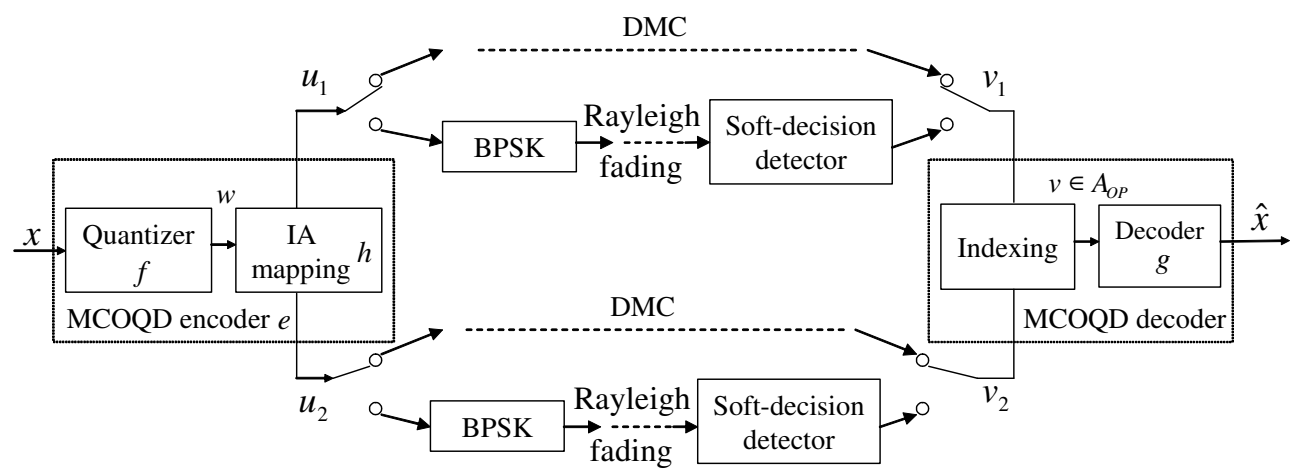

Fig. 1. System model of MCOQD over two DMCs and Rayleigh fading channels.

A system block diagram of MCOQD for two DMCs is shown in Fig. 1 (the upper channel branch). A source sample $x$ is first quantized with an $M$-level scalar quantizer, which maps the source $x \in \mathbb{R}$ into one of $M$ values. The quantization effects an encoder mapping $f: \mathbb{R} \rightarrow\{1,2, \ldots, M\}$, whose output is quantizer index $w$, i.e., $w=f(x)$. $w$ is mapped to two descriptions $u_{1}$ and $u_{2}$, at bit rates of $R_{1}$ bits and $R_{2}$ bits, respectively, with $2^{R_{1}+R_{2}} \geq M$. The mapping $h:\{1,2, \ldots, M\} \rightarrow\left\{1, \ldots, 2^{R_{1}}\right\} \times\left\{1, \ldots, 2^{R_{2}}\right\}$ realizes a multiple-description IA [3]. Each channel is an independent discrete memoryless channel (DMC) with erasure output. Symbol erasure is treated as a channel output, denoted by " $E$ " in the DMC output alphabet. Each distinct channel output pair $\left(v_{1}, v_{2}\right)$ is assigned a unique index value $v \in A_{O P}=\{1, \ldots, L\}$. The decoder effects a mapping $g: A_{O P}=\{1, \ldots, L\} \rightarrow \mathbb{R} . v$ is decoded to one of the $L$ possible values in a decoder codebook $\mathcal{C}=\left\{\tilde{x}_{1}, \ldots, \tilde{x}_{L}\right\} \subset \mathbb{R}$, i.e., $\hat{x}=g(v)$. Considering all possible channel output values, the decoder codebook size is therefore $L=\left(2^{R_{1}}+1\right)\left(2^{R_{2}}+1\right)$.

The above formulation of the encoder involves two mappings: encoder mapping $f$ and IA mapping $h$. However, the encoder is treated differently in MCOQD. MCOQD seeks to directly optimize the mapping from $x$ to $\left(u_{1}, u_{2}\right)$, instead of the two consecutive mappings $f$ and $h$. We define an equivalent encoder mapping $e: \mathbb{R} \rightarrow\left\{1, \ldots, 2^{R_{1}}\right\} \times\left\{1, \ldots, 2^{R_{2}}\right\}$. MCOQD chooses an encoder $e$ that always picks the best output $\left(u_{1}, u_{2}\right)$.

Akin to the generalized Lloyd algorithm (GLA) [8], the MCOQD algorithm [7] is an iterative improvement algorithm constructed based on two necessary optimality conditions. The nearest neighbor condition gives the optimal $e$ for fixed $g$, and the centroid condition gives the optimal $g$ for fixed $e$. Let each channel input pair $\left(u_{1}, u_{2}\right)$ be assigned a unique index $u \in A_{I P}=\{1, \ldots, M\}$. The optimal encoder $e^{*}$, for a fixed decoder $g$, selects the best channel input symbol $u^{*} \in A_{I P}$ to minimize the expected squared error distortion, i.e.,

$$
u^{*}(x)=\arg \min _{i \in A_{I P}} \sum_{j=1}^{L} P(v=j \mid u=i)\left(x-\tilde{x}_{j}\right)^{2}
$$

where $P(v=j \mid u=i)$ is the probability that the multiple-channel output symbol $v=j \in A_{O P}$, given the multiple-channel input symbol $u=i \in A_{I P}$, with $u=i \Longleftrightarrow\left(u_{1}, u_{2}\right)=\left(i_{1}, i_{2}\right)$ and $v=j \Longleftrightarrow$ $\left(v_{1}, v_{2}\right)=\left(j_{1}, j_{2}\right)$. The channels are independent so that the multiple-channel symbol transition probabilities can be written in terms of the single-channel symbol transition probabilities as:

$$
P(v=j \mid u=i)=P\left(v_{1}=j_{1} \mid u_{1}=i_{1}\right) P\left(v_{2}=j_{2} \mid u_{2}=i_{2}\right) .
$$

The optimal decoder $g^{*}$, for a fixed encoder $e$, maps $j \in A_{O P}$ to $\tilde{x}_{j}^{*} \in \mathcal{C}^{*}$, where

$$
\tilde{x}_{j}^{*}=\frac{\sum_{i=1}^{M} E\left[X \mid X \in Q_{i}\right] P\left(X \in Q_{i}\right) P(v=j \mid u=i)}{\sum_{i=1}^{M} P\left(X \in Q_{i}\right) P(v=j \mid u=i)},
$$

and $Q_{i}$ is the set of all source values encoded to $u=$ $i$. MCOQD offers several advantages over the MDSQ design in [3]. MCOQD is simpler, can handle both erasure and symbol errors, and is readily extended to designing for more than two descriptions or channels.

\section{MCOQD OVER RAYLEIGH FADING CHANNELS}

We consider multiple description communication over two independent Rayleigh channels (the lower channel branch in Fig. 1) with a MCOQD encoder at the transmitter and a MCOQD decoder at the receiver. The two bitstreams generated by the MCOQD encoder are 
BPSK modulated and sent over two Rayleigh fading channels. Specifically, each bit of the binary $R_{i}$-tuple, $u_{i} \in\left\{1, \ldots, 2^{R_{i}}\right\}$, is modulated to a BPSK signal. Let $S_{i}\left(S_{i} \in\left\{\sqrt{E_{b}},-\sqrt{E_{b}}\right\}, i=1,2\right), W_{i}$, and $Y_{i}$ be the transmitted BPSK signal, channel noise, and the received signal, respectively, of the $i$-th channel, then

$$
Y_{i}=H_{i} S_{i}+W_{i}, \quad i=1,2
$$

where $W_{i}$ is Gaussian and $H_{i}$ is Rayleigh distributed, i.e.,

$$
P_{H_{i}}\left(h_{i}\right)=\frac{h_{i}}{\sigma_{i}^{2}} e^{-h_{i}^{2} / 2 \sigma_{i}^{2}}, h_{i} \geq 0 .
$$

We use soft-decision detectors at the receiver, as shown in Fig. 1. Suppose the detector for channel $i$ uses $N_{i}$ detection regions. The concatenation of the BPSK modulator, the Rayleigh fading channel and the soft detector for each channel can be modelled as a binary-input and $N_{i}$-output DMC. For channel $i$ with rate $R_{i}$, the binary-input, $N_{i}$-output $\mathrm{DMC}$ is used $R_{i}$ times. Suppose $\left(u_{i}^{1}, \ldots, u_{i}^{R_{i}}\right)$ is sent and $\left(v_{i}^{1}, \ldots, v_{i}^{R_{i}}\right)$ is received. Assuming the DMC is memoryless from one use to another, the transition probabilities for the $R_{i}$ tuple input channel can be calculated as

$$
P\left(v_{i} \mid u_{i}\right)=\prod_{l=1}^{R_{i}} P\left(v_{i}^{l} \mid u_{i}^{l}\right)
$$

where $P\left(v_{i}^{l} \mid u_{i}^{l}\right)$ is a transition probability of the binaryinput, $N_{i}$-output DMC. In practice, independent statistics for successive channel use can be achieved using bitinterleaving. With (5), the multiple-channel transition probability $P(v \mid u)$ can be calculated using (2) and used as the channel parameters for MCOQD.

\section{A. Soft-detector with three detection regions}

In [5], the MDSQ in [3] is applied to Rayleigh fading channels, where a channel is declared to be in a working state or erasure state by comparing the fading parameter with a predetermined threshold. In this paper, channel erasure is deemed to occur when the energy of the received signal is not large enough. To this end we use a soft-decision detector with three detection regions. Specifically, the detector outputs symbols "0", "E" or " 1 " for detection regions $\left(-\infty,-T_{i}\right),\left[-T_{i}, T_{i}\right]$ and $\left(T_{i}, \infty\right)$, respectively, with " $E$ " standing for "erasure". The equivalent binary-input ternary-output DMC is shown in Fig. 2 with bit error probability $\epsilon_{i}$ and erasure probability $\gamma_{i}$.

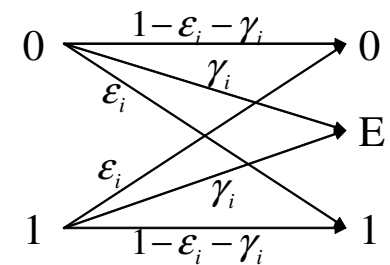

Fig. 2. The binary-input ternary-output DMC with bit error probability $\epsilon_{i}$ and erasure probability $\gamma_{i}$.

For a given $H_{i}=h_{i}$, the conditional $\epsilon_{i}$ and $\gamma_{i}$ can be evaluated as

$$
\begin{aligned}
& \epsilon_{i}\left(T_{i} \mid H_{i}=h_{i}\right)=Q\left(\frac{T_{i}+h_{i} \sqrt{E_{b}}}{\sqrt{W_{0} / 2}}\right) \\
& \gamma_{i}\left(T_{i} \mid H_{i}=h_{i}\right)=Q\left(\frac{-T_{i}+h_{i} \sqrt{E_{b}}}{\sqrt{W_{0} / 2}}\right)-Q\left(\frac{T_{i}+h_{i} \sqrt{E_{b}}}{\sqrt{W_{0} / 2}}\right)
\end{aligned}
$$

where $W_{0}$ is single-sided noise power spectrum density. By averaging $\epsilon_{i}\left(T_{i} \mid H_{i}=h_{i}\right)$ and $\gamma_{i}\left(T_{i} \mid H_{i}=h_{i}\right)$ over the probability density function of $H_{i}$, we obtain

$$
\begin{aligned}
\epsilon_{i}\left(T_{i}\right)= & \int_{0}^{\infty} \frac{h_{i}}{\sigma_{i}^{2}} e^{-h_{i}^{2} / 2 \sigma_{i}^{2}} Q\left(\frac{T_{i}+h_{i} \sqrt{E_{b}}}{\sqrt{W_{0} / 2}}\right) d h_{i}, \\
\gamma_{i}\left(T_{i}\right)= & \int_{0}^{\infty} \frac{h_{i}}{\sigma_{i}^{2}} e^{-h_{i}^{2} / 2 \sigma_{i}^{2}}\left[Q\left(\frac{-T_{i}+h_{i} \sqrt{E_{b}}}{\sqrt{W_{0} / 2}}\right)\right. \\
& \left.-Q\left(\frac{T_{i}+h_{i} \sqrt{E_{b}}}{\sqrt{W_{0} / 2}}\right)\right] d h_{i} .
\end{aligned}
$$

The end-to-end distortion, $D$, is determined by the multiple-channel symbol transition probabilities $P(v \mid u)$. Variation of the thresholds $T_{i}$ affects $P(v \mid u)$ and thus $D$. Our objective is to determine the thresholds $T_{i}$ such that $D$ is minimized:

$$
\left[T_{1}, T_{2}\right]_{\text {opt }}=\arg \min _{T_{1}, T_{2}} D\left[\epsilon_{1}\left(T_{1}\right), \epsilon_{2}\left(T_{2}\right), \gamma_{1}\left(T_{1}\right), \gamma_{2}\left(T_{2}\right)\right] .
$$

$D$ is obtained by running the MCOQD algorithm for the channels with multiple-channel symbol transition probabilities determined by $\epsilon_{i}\left(T_{i}\right)$ and $\gamma_{i}\left(T_{i}\right)$.

\section{B. Soft-decision detectors with more than three detection regions}

In [6], COQD is applied to a Rayleigh fading channel with a soft-decision detector at the receiver. The numerical results show that the performance improves as the number of detection regions increases. We note that the notion of channel "erasure" becomes less relevant as the number of the detection regions increases beyond three. 
In Fig. 1, the output $Y_{i}$ of the $i$-th Rayleigh fading channel is detected through the use of a uniform scalar quantizer with $N_{i}>3$ quantization regions. Let the step size of the uniform scalar quantizer be $\Delta_{i}$. The detection thresholds satisfy

$$
T_{i}^{j}= \begin{cases}-\infty, & j=-1 \\ \left(j+1-\frac{N_{i}}{2}\right) \Delta_{i}, & j=0,1, \ldots, N_{i}-2 \\ \infty, & j=N_{i}-1 .\end{cases}
$$

For $N_{i}=3$, the thresholds $T_{i}^{0}=-\Delta_{i} / 2$ and $T_{i}^{1}=$ $\Delta_{i} / 2$ specialize to the detection thresholds defined in section III-A. The problem of finding optimal detection thresholds $T_{i}^{j}$ is equivalent to choosing an optimal step size $\Delta_{i}$ for a fixed $N_{i}$. Following the approach in section III-A, channel symbol transition probabilities can be calculated as a function of $\Delta_{i}$, and placed into a transition probability matrix denoted as $\pi_{i}$. The optimal step sizes are obtained as

$$
\left[\Delta_{1}, \Delta_{2}\right]_{\text {opt }}=\arg \min _{\Delta_{1}, \Delta_{2}} D\left[\pi_{1}\left(\Delta_{1}\right), \pi_{2}\left(\Delta_{2}\right)\right] .
$$

One key difference between our optimization approach and [6] is that we choose $\Delta_{i}$ to minimize the end-toend distortion while [6] chooses to maximize the mutual information between the binary-input and soft-decision output of the DMC. The $\Delta_{i}$ obtained using the approach in [6] is suboptimal for our objective of minimizing endto-end distortion.

\section{Adaptive MCOQD in the presence of channel mis- match}

The designed MCOQD encoder and decoder in sections III-A and III-B are sensitive to the channel parameters, i.e., the Rayleigh fading parameter $\sigma_{i}$ and the noise parameter $W_{0}$ in (8) and (9). The channel parameters can be estimated at the receiver and sent back to the transmitter for the design purpose. However, a feedback channel may not be available in practice. It is of particular interest to study the case where the actual channel parameters differ from those used for the design.

We consider a scenario wherein channel statistics are slowly varying. The decoder has perfect knowledge of the channels, i.e., $\sigma_{i}$ and $W_{0}$, and adapts accordingly, while the encoder has no knowledge of the varying channels and therefore fixed. The MCOQD encoder and decoder and the soft-decision detector are initially designed based on predetermined channel parameters, using the methods introduced in sections III-A and IIIB. When applied to the channels, both the detector and decoder adapt given up-to-date knowledge of $\sigma_{i}$ and $W_{0}$. The decoder recomputes the reproductions according to
(3), given the fixed encoder partition, and the channel transition probabilities are calculated based on reoptimized detection thresholds.

\section{PERformance Results}

We first simulate the performance of MCOQD where the channel parameters used for design match the actual channel parameters in operation. A memoryless Gaussian and a memoryless Laplacian source with zero mean and unit variance are considered. For simplicity, we assume that the two Rayleigh fading channels have identical fading statistics, and thus the detection thresholds for the two channels are chosen to be equal, i.e., $T_{1}=T_{2}=T$ in section III-A and $\Delta_{1}=\Delta_{2}=\Delta$ in section III-B. Let $\delta=\Delta / \sqrt{E_{b}}$ be a normalized step size. Performance is expressed as signal-to-distortion ratio (SDR) in $\mathrm{dB}$ versus average channel SNR measured at the receiver of one channel, i.e., SNR $=\frac{E_{b}}{W_{0}} E\left\{H^{2}\right\}=\frac{2 \sigma E_{b}}{W_{0}}$.

The performance of the MCOQD system and the associated optimized $\delta$ are shown in Fig. 3 and Table I, respectively. Table I shows that the optimized detector step size decreases as the channel SNR or the number of detection regions increases. Fig. 3 shows the performance of MCOQD improving as the number of detection regions increases, e.g., an improvement of 0.2 $0.3 \mathrm{~dB}$ is achieved from 3 to 10 detection regions. The performance of MDSQ with an optimized 3-level softdetector is also included for comparison in Fig. 3. The Lagrange parameter values needed to perform MDSQ design are determined using the erasure probabilities $\gamma_{1}$ and $\gamma_{2}$ [7]. MCOQD is seen to perform better than MDSQ for all channel SNRs. The gain is due to the fact that MCOQD provisions for channel symbol errors whereas MDSQ does not.

\begin{tabular}{|c|c|c|c|c|c|c|}
\hline \multirow{2}{*}{$\begin{array}{c}\text { SNR } \\
(\mathrm{dB})\end{array}$} & \multicolumn{3}{|c|}{ Gaussian } & \multicolumn{3}{c|}{ Laplacian } \\
\cline { 2 - 7 } & $N=3$ & $N=4$ & $N=10$ & $N=3$ & $N=4$ & $N=10$ \\
\hline 0 & 0.84 & 0.62 & 0.28 & 0.74 & 0.53 & 0.24 \\
\hline 1 & 0.78 & 0.54 & 0.22 & 0.61 & 0.50 & 0.20 \\
\hline 2 & 0.66 & 0.46 & 0.20 & 0.55 & 0.41 & 0.18 \\
\hline 3 & 0.56 & 0.39 & 0.19 & 0.45 & 0.39 & 0.16 \\
\hline 4 & 0.43 & 0.33 & 0.16 & 0.41 & 0.33 & 0.14 \\
\hline 5 & 0.36 & 0.31 & 0.15 & 0.39 & 0.31 & 0.13 \\
\hline 6 & 0.33 & 0.25 & 0.14 & 0.33 & 0.25 & 0.12 \\
\hline 7 & 0.29 & 0.22 & 0.11 & 0.29 & 0.22 & 0.10 \\
\hline 8 & 0.26 & 0.19 & 0.09 & 0.28 & 0.21 & 0.07 \\
\hline
\end{tabular}

TABLE I

OPTIMIZED DETECTOR STEP SIZE $\delta=\frac{\Delta}{\sqrt{E_{b}}}$ FOR THE NUMBER OF DETECTION REGIONS $N_{1}=N_{2}=N$

We compare the performance of regular MCOQD, with fixed encoder and decoder, and MCOQD with 


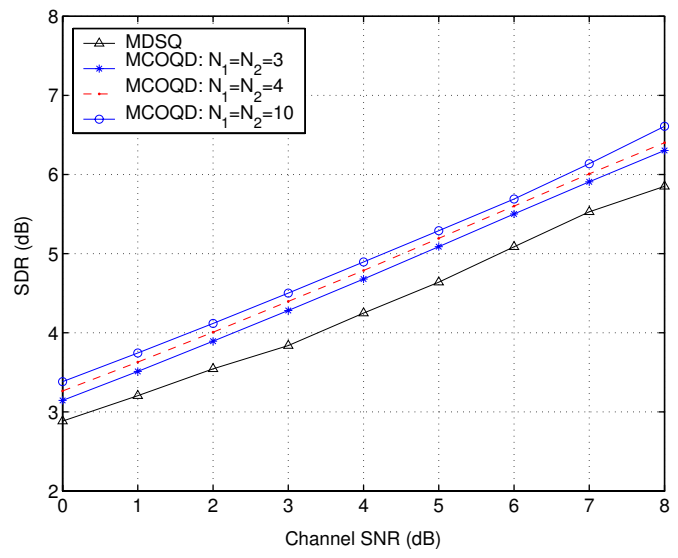

(a)

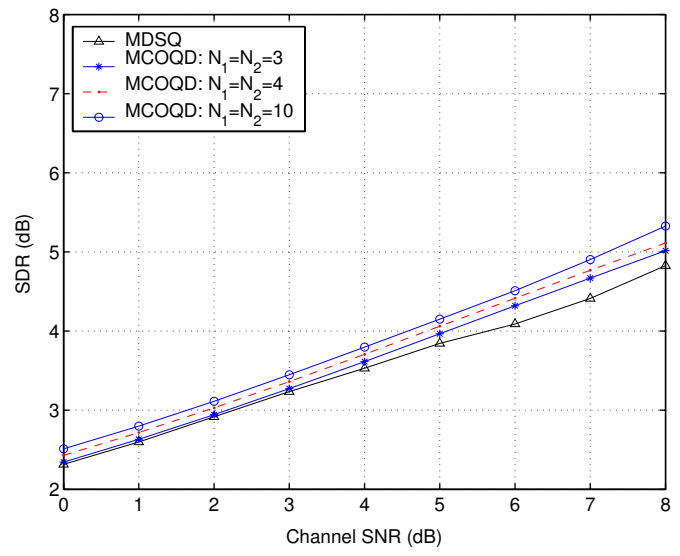

(b)

Fig. 3. Performance of MDSQ and MCOQD with $R_{1}=R_{2}=1$ bit per source sample per Rayleigh fading channel (bpss/channel). (a) Memoryless Gaussian source. (b) Memoryless Laplacian source.

adaptive decoder and fixed encoder. Fig. 4 shows that the improvement of adaptive MCOQD over regular MCOQD increases with channel mismatch. At $8 \mathrm{~dB}$ actual channel $\mathrm{SNR}$, for instance, the MCOQD designed for $8 \mathrm{~dB}$ SNR outperforms the MCOQD designed for $0 \mathrm{~dB}$ SNR by 1.1 $\mathrm{dB}$ SDR. Adaptive MCOQD deduces this performance gap by $0.3 \mathrm{~dB}$. Hence, the bulk of the performance gap can be attributed to encoder mismatch.

\section{REFERENCES}

[1] V.K. Goyal, "Multiple description coding: compression meets the network," IEEE Sig. Proc. Mag., vol. 18, pp. 74-93, Sept. 2001.

[2] N. Farvardin and V. Vaishampayan, "Optimal quantizer design for noisy channels: an approach to combined source-channel coding," IEEE Trans. Inform. Theory, vol. 33, no. 6, pp. 827838, Nov. 1987.

[3] V. Vaishampayan, "Design of Multiple Description Scalar Quantizers," IEEE Trans. Inform. Theory, vol. 39, no. 3, pp. 821834, May. 1993.

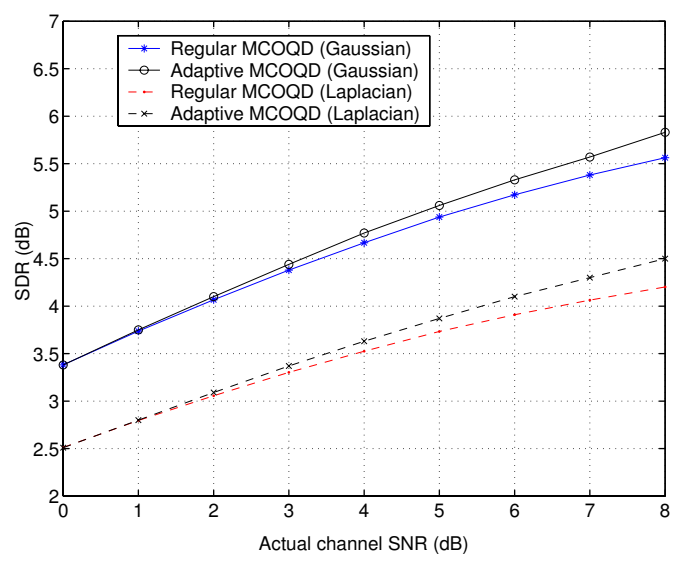

(a)

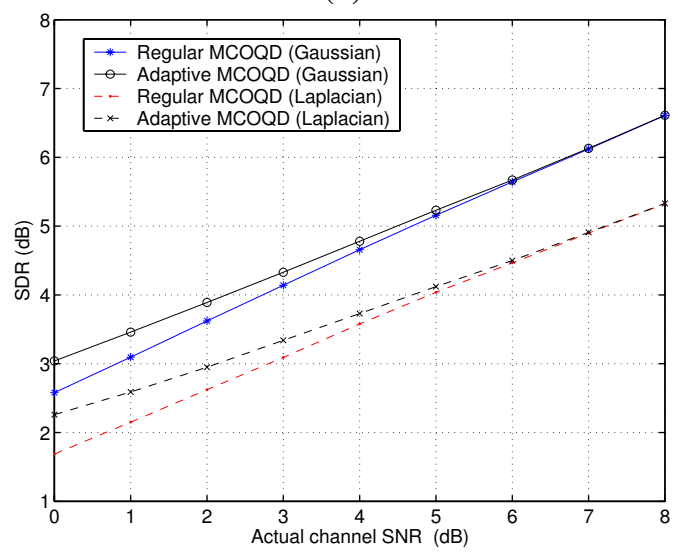

(b)

Fig. 4. Performance of regular MCOQD, and MCOQD with an adaptive detector and decoder, for the Gaussian and Laplacian source, over Rayleigh fading channels. $R_{1}=R_{2}=1 \mathrm{bpss} / \mathrm{channel}$ and $N_{1}=N_{2}=10$. (a) Design channel SNR $=0 \mathrm{~dB}$. (b) Design channel $\mathrm{SNR}=8 \mathrm{~dB}$.

[4] N. Görtz and P. Leelapornchai, "Optimization of the index assignments for multiple description vector quantizers," IEEE Trans. Commun., vol. 51, no. 3, pp. 336-340, Mar. 2003.

[5] S. Yang and V. Vaishampayan , "Low-delay communication for Rayleigh fading channels: An application of the multiple description quantizer," IEEE Trans. Commun., vol. 43, no. 11, pp. 2771-2783, Nov. 1995.

[6] F. Alajaji and N. Phamdo, "Soft-Decision COVQ for RayleighFading Channels," IEEE Commun. Letters, vol. 2, pp. 162-164, June 1998.

[7] Y. Zhou and W.-Y. Chan, "Multiple description quantizer design using a channel optimized quantizer approach," 38th Conf. Inform. Science and Systems (CISS 2004), Princeton, NJ, March, 2004.

[8] Y. Linde, A. Buzo, and R.M. Gray, "An algorithm for vector quantizer design," IEEE Trans. Commun., vol. 28, no. 1, pp. 8495, Jan. 1980. 